\title{
Charge-transfer excited states in the donor/ acceptor interface from large-scale GW calculations
}

Cite as: J. Chem. Phys. 151, 114109 (2019); https://doi.org/10.1063/1.5113944

Submitted: 10 June 2019 . Accepted: 13 August 2019 . Published Online: 18 September 2019

Takatoshi Fujita (D), Yoshifumi Noguchi (D), and Takeo Hoshi (D)
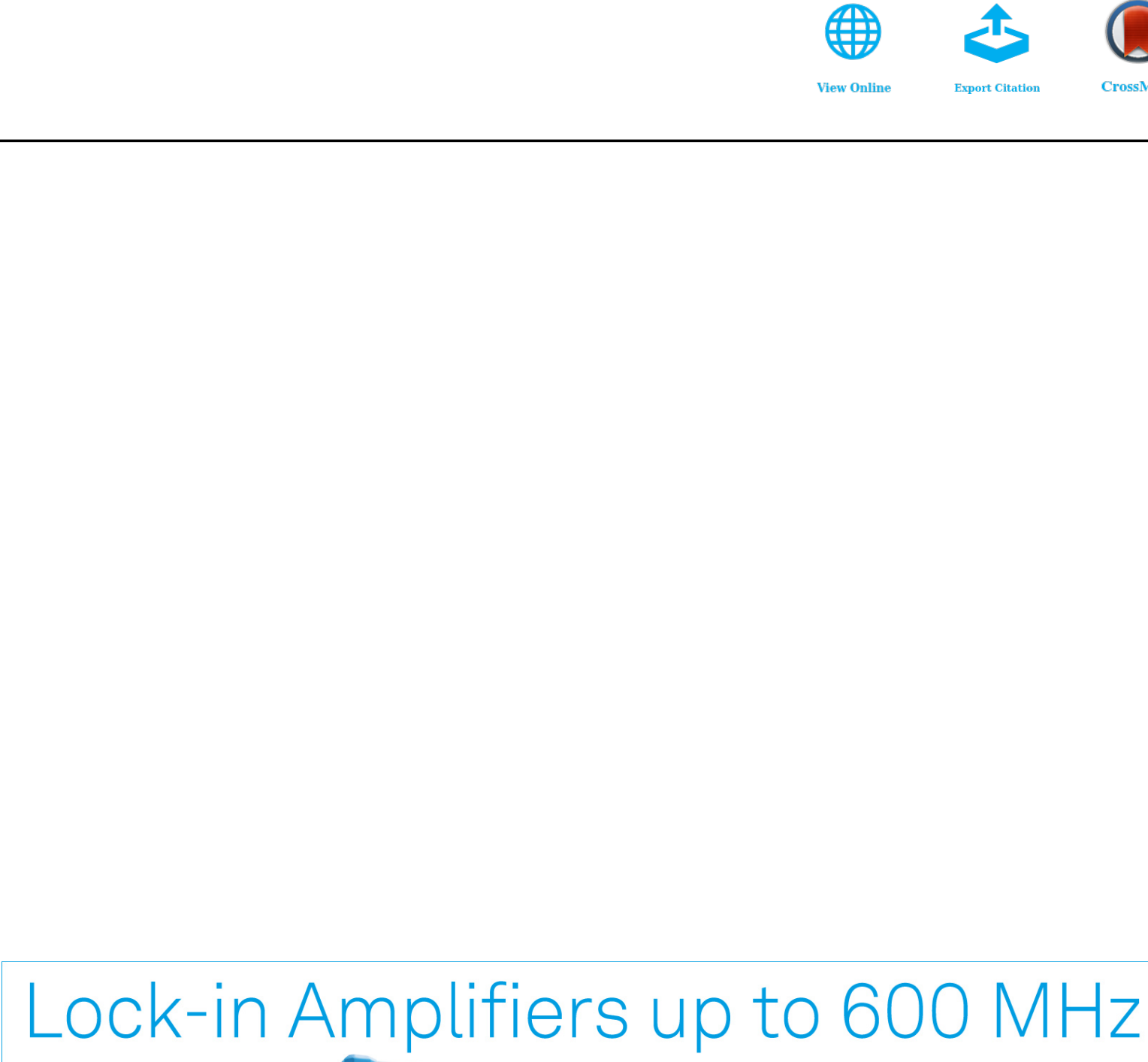

starting at $\$ 6,210$ 


\title{
Charge-transfer excited states in the donor/acceptor interface from large-scale GW calculations
}

\author{
Cite as: J. Chem. Phys. 151, 114109 (2019); doi: 10.1063/1.5113944 \\ Submitted: 10 June 2019 • Accepted: 13 August 2019 • \\ Published Online: 18 September 2019
}

\begin{abstract}
Takatoshi Fujita, , a) (Doshifumi Noguchi, ${ }^{2}$ (D) and Takeo Hoshi ${ }^{3}$

\section{AFFILIATIONS}

${ }^{1}$ Institute for Molecular Science, Okazaki, Aichi 444-0865, Japan

2Department of Applied Chemistry and Biochemical Engineering, Graduate School of Engineering, Shizuoka University, Hamamatsu, Shizuoka 432-8561, Japan

${ }^{3}$ Department of Applied Mathematics and Physics, Tottori University, Tottori 680-8550, Japan
\end{abstract}

a)Electronic mail: tfujita@ims.ac.jp

\begin{abstract}
Predicting the charge-transfer (CT) excited states across the donor/acceptor (D/A) interface is essential for understanding the charge photogeneration process in an organic solar cell. Here, we present a fragment-based GW implementation that can be applied to a D/A interface structure and thus enables accurate determination of the CT states. The implementation is based on the fragmentation approximation of the polarization function and the combined GW and Coulomb-hole plus screened exchange approximations for self-energies. The fragmentbased GW is demonstrated by application to the pentacene/ $\mathrm{C}_{60}$ interface structure containing more than 2000 atoms. The $\mathrm{CT}$ excitation energies were estimated from the quasiparticle energies and electron-hole screened Coulomb interactions; the computed energies are in reasonable agreement with experimental estimates from the external quantum efficiency measurements. We highlight the impact of the induced polarization effects on the electron-hole energetics. The proposed fragment-based GW method offers a first-principles tool to compute the quasiparticle energies and electronic excitation energies of organic materials.
\end{abstract}

Published under license by AIP Publishing. https://doi.org/10.1063/1.5113944

\section{INTRODUCTION}

Charge-transfer (CT) excited states formed across the donor/ acceptor (D/A) interface play a key role in the charge photogeneration process in organic photovoltaics. ${ }^{1,2}$ The charge-separation and charge-recombination rates largely depend on the nature of the CT states. Moreover, the energy of the CT state correlates with the open-circuit voltage value in an organic solar cell. ${ }^{3,4}$ Therefore, understanding the energetics and nature of CT states and correlating them with interfacial morphologies ${ }^{5}$ are essential. Theoretical investigations on organic/organic interfaces have provided microscopic information regarding the energies and wave function properties of the CT states. ${ }^{6-9}$ Nevertheless, first-principles computations of the CT states in an organic/organic interface remain challenging because both electron correlation and polarizable environmental effects must be incorporated.
One of the most practically useful excited-state methods for a large system is the time-dependent density functional theory (TDDFT). ${ }^{10,11}$ However, TDDFT cannot provide the correct $-1 / R$ asymptotic behaviors for CT states, ${ }^{12}$ where $R$ denotes the separation between the donor and acceptor molecules. In addition, the TDDFT tends to underestimate the CT energies ${ }^{13}$ in large molecules or a molecular cluster. The long-range correction scheme ${ }^{14,15}$ can remedy the failure of TDDFT, and long-range corrected functionals ${ }^{16-19}$ can yield more reasonable descriptions for the CT states. As well as a quantum mechanical method for excited states, the effect of polarizable environments must also be considered. The considerable charge-density difference between the CT and ground states induces polarization of the surrounding molecules, thereby resulting in screening of the electron-hole $(\mathrm{e}-\mathrm{h})$ Coulomb interaction. Therefore, successful prediction of the CT states requires the treatment of state-specific polarization 
effects, ${ }^{20,21}$ which goes beyond the standard electrostatic embedding scheme.

The GW many-body Green's function theory ${ }^{22-24}$ can offer an accurate and practical scheme to explore electronic states in a condensed phase. The quasiparticle energy directly corresponds to a charged excitation energy, such as an ionization potential or an electron affinity. The neutral excitation energy can also be computed in combination with the Bethe-Salpeter equation (BSE). ${ }^{25,26}$ The GW+BSE approach ${ }^{27,28}$ describes the e-h interaction as the screened Coulomb potential; therefore, it is expected to be a promising tool for computing the CT states in polarizable environments. Recently, several attempts have been made to extend the applicability of GW to larger and more complex systems. Owing to the development of computational algorithms, performing a GW calculation for a system containing more than 100 atoms has become possible. $^{29-32}$ The polarizable continuum model ${ }^{33,34}$ and the quantum mechanical/molecular mechanics $(\mathrm{QM} / \mathrm{MM})^{35-37}$ approach have been combined, allowing for applications to molecules in complex environments. In particular, Li et al. ${ }^{35,36}$ have developed the QM/MM method merging the GW formalism with the polarizable model. Another possible approach toward complex systems is to utilize a fragment-based electronic structure method, ${ }^{38,39}$ which approximates the energy or wave functions of an entire system from subsystem calculations. On the basis of a fragmentbased method, ${ }^{40}$ Fujita and Noguchi ${ }^{41}$ have developed the largescale Green's function method within the static Coulomb-hole plus screened exchange (COHSEX) approximation and reported the static COHSEX calculation for a system containing more than 1000 atoms.

In this manuscript, we report on the implementation of GW based on the fragment molecular orbital (FMO ${ }^{40}$ method and the applications to a realistic molecular structure of a D/A bilayer heterojunction containing more than 2000 atoms. The implementation is based on the approximation of the polarization function by fragment molecular orbitals (MOs) and combined GW and static COHSEX approximations for self-energies. We explore the quasiparticle energies and CT states in the D/A interface and investigate the environmental polarization effects on the electronic states.

\section{METHOD}

We first summarize Hedin's equations for evaluating selfenergies. $^{22}$ The GW self-energy for an imaginary frequency axis is given by the following set of equations: ${ }^{42}$

$$
\begin{gathered}
\sum\left(r_{1}, r_{2}, i \omega\right)=-\frac{1}{2 \pi} \int d \omega^{\prime} G\left(r_{1}, r_{2}, i \omega+i \omega^{\prime}\right) W\left(r_{1}, r_{2}, i \omega^{\prime}\right), \\
G\left(r_{1}, r_{2}, i \omega\right)=\sum_{p}\left[\frac{\psi_{p}\left(r_{1}\right) \psi_{p}^{*}\left(r_{2}\right)}{i \omega-\epsilon_{p}+E_{F}}\right], \\
W\left(r_{1}, r_{2}, i \omega\right)=v\left(r_{1}, r_{2}\right)+\int d r_{3} d r_{4} v\left(r_{1}, r_{3}\right) \chi_{0}\left(r_{3}, r_{4}, i \omega\right) \\
\quad \times W\left(r_{4}, r_{2}, i \omega\right),
\end{gathered}
$$

$$
\chi_{0}\left(r_{1}, r_{2}, i \omega\right)=\sum_{\text {spins }} \sum_{i, a}\left[\frac{\psi_{i}^{*}\left(r_{1}\right) \psi_{a}\left(r_{2}\right) \psi_{a}^{*}\left(r_{2}\right) \psi_{i}\left(r_{1}\right)}{i \omega-\epsilon_{i}+\epsilon_{a}}+\text { c.c. }\right] .
$$

Here, $G$ is the Green's function, $W$ is the screened Coulomb potential at the random-phase approximation level, $v$ is the bare Coulomb potential, $\chi_{0}$ is the noninteracting density-response function or irreducible polarizability, and $E_{F}$ is the Fermi level. Indices $i, a$, and $p$ refer to an occupied, virtual, and generic MO, respectively.

We aim to approximate the GW self-energies within the FMO method. ${ }^{43}$ We summarize the fragment-based self-energy calculations developed in the earlier study. ${ }^{41}$ In the FMO method, an entire system is divided into small subsystems referred to as fragments, and the $\mathrm{MO}$ calculations are performed for the fragment monomers and fragment dimers. For example, the solution of the Fock matrix of a fragment monomer yields the MOs localized within the fragment, the monomer MOs $\left(\psi_{p}^{I}\right)$, and associated orbital energies $\left(\epsilon_{p}^{I}\right)$. Hereafter, we use $I$ and $J$ as fragment indices. The FMO-based GW implementation adapts the monomer MOs to approximate the polarization function and screened Coulomb potential. ${ }^{41}$ This is in contrast to a standard GW implementation in which the input orbitals for the polarization function are delocalized over an entire system. The total polarization function is approximated as the sum of intrafragment polarization functions, $\chi_{0}=\sum_{J} \chi_{0}^{J}$. Here, $\chi_{0}^{J}$ is an intrafragment polarization function of fragment $J$ that is calculated from the localized MOs in the FMO method,

$$
\chi_{0}^{J}\left(r_{1}, r_{2}, i \omega\right)=\sum_{\text {spins }} \sum_{i, a \in J}\left[\frac{\psi_{i}^{J *}\left(r_{1}\right) \psi_{a}^{J}\left(r_{2}\right) \psi_{a}^{J *}\left(r_{2}\right) \psi_{i}^{J}\left(r_{1}\right)}{i \omega-\epsilon_{i}^{J}+\epsilon_{a}^{J}}+c . c .\right] .
$$

Note that $\chi_{0}^{J}$ describes the polarization within a fragment $J$, i.e., both occupied and virtual MOs belong to the same fragment. Therefore, the fragmentation approximation neglects the interfragment polarization terms and the delocalization of MOs. The screened Coulomb potential is obtained from the approximated polarization function. The self-energies are calculated from a fragment Green's function and the screened Coulomb potential, $\Sigma=1 / 2 \pi \int d \omega^{\prime} G^{I} W$. The accuracy of the approximated screened Coulomb potential has been confirmed for the molecular aggregates.

Herein, we propose a hybrid computational scheme for selfenergies, combining the GW and static COHSEX approximations. The static COHSEX approximation is the static limit of GW and tend to overestimate fundamental gaps. Although evaluating the dynamical-part of self-energy is inevitable, calculating the dynamically screened Coulomb potential of an entire system is still computationally too demanding even when the fragment-based approximation is used. Here, we introduce the hybrid scheme, where the dynamically screened Coulomb potential is explicitly evaluated for a target fragment, while the statically screened Coulomb potential of the entire system is evaluated at the static COHSEX level. A similar approach has also been used in the aforementioned QM/MM method proposed by Li et al., ${ }^{35,36}$ in which the GW self-energy is defined as the gas-phase GW self-energy plus a polarization energy of the QM/MM total system obtained at the static COHSEX level. On the basis of this idea, we write the FMO-GW self-energy as follows:

$$
\Sigma_{F M O-G W, p}^{I}=\Sigma_{G W^{I}, p}^{I}+\Sigma_{C O H S E X, p}^{I}-\Sigma_{C O H S E X^{I}, p}^{I} .
$$


Here, $\Sigma_{C O H S E X, p}^{I}$ is calculated from the total screened Coulomb potential; it includes the induced polarization energy of an entire system on electron addition or removal in fragment $I$. On the contrary, $\Sigma_{G W^{I}, p}^{I}$ or $\Sigma_{C O H S E X^{I}, p}^{I}$ is the GW or static COHSEX self-energy that is defined by the fragment Green's function and a fragment screened Coulomb potential, $\Sigma=1 / 2 \pi \int d \omega^{\prime} G^{I} W^{I}$. Note that $W^{I}$ is obtained by the intrafragment density-response function of a target fragment, $W^{I}=v+\int d r_{3} d r_{4} v \chi^{I} W^{I}$, without the polarization functions of the other fragments. Therefore, $\Sigma_{G W^{I}, p}^{I}$ or $\Sigma_{C O H S E X^{I}, p}^{I}$ does not include the induced polarization energy of the surrounding fragments. The expression denotes that electron correlation and orbital relaxation within a target fragment are treated at the GW level, whereas the associated induced polarization energy of an entire system is computed at the static COHSEX level. We have shown that the static COHSEX can appropriately describe the solid-state effects on electronic states ${ }^{9}$ and expect that the combined GW and static COHSEX approach can provide a reasonable approximation for a weakly interacting molecular system. The FMO-GW quasiparticle energy of the fragment is calculated in a perturbation manner as follows:

$$
\epsilon_{G W, p}^{I}=\epsilon_{H F / K S, p}^{I}+Z_{p}\left[\Sigma_{F M O-G W, p}^{I}-V_{p}^{X C}\right]
$$

where $\epsilon_{H F / K S, p}^{I}$ is the Hartree-Fock (HF) or Kohn-Sham (KS) orbital energy, $Z_{p}$ is the renormalization factor, and $V_{p}^{X C}$ is the HF exchange potential or an exchange-correlation potential.

The environmental effects incorporated in the present method are now discussed in more detail. In the FMO-HF or FMO-DFT method, ${ }^{40,43}$ self-consistent field calculations are repeated for fragment monomers until all the fragment charges have converged; thus, the many-body polarization of the ground state can be effectively taken into account. By contrast, the static COHSEX selfenergy term $\left(\Sigma_{C O H S E X, p}^{I}\right)$ represents electronic relaxation of all the fragments that is induced by the formation of the charged excitation in some fragment. Therefore, the FMO-COHSEX describes the polarization of charged states. Earlier studies ${ }^{44,45}$ have discussed that the molecular packing effect on ionization potential or the electron affinity can be divided into the electrostatic and induced polarization energies. The former represents the interaction between a charge and multipole moments of the surrounding molecules, while the latter describes the interaction between a charge and induced dipoles. In the FMO calculation, the electrostatic effect can be considered by the HF or DFT level, and the induced polarization effect can be incorporated by the static COHSEX level.

Our GW implementation relies on the Gaussian atomic orbital basis and the auxiliary functions. ${ }^{42,46}$ The auxiliary functions and the three-center electron-repulsion integrals, which are necessary to evaluate self-energies, ${ }^{42}$ were obtained from the Cholesky decomposition with an adaptive metric (CDAM) technique developed by Okiyama et al. ${ }^{47}$ According to Ren et al. ${ }^{42}$ and Wilhelm et al., ${ }^{48}$ evaluation of the GW self-energy is based on integration along the imaginary. The FMO-GW method was implemented into the locally modified version of the ABINIT-MP program. ${ }^{49-51}$ Further details of the implementation, as well as the working equations for FMO-GW self-energies, are given in the supplementary material.

\section{RESULTS AND DISCUSSION}

\section{A. Accuracy of FMO-GW self-energy}

In this section, we briefly discuss the accuracy of the FMO-GW self-energies of a fragment monomer. The present implementation is based on the fragmentation approximation of the polarization function and the combined static COHSEX and GW approximation which neglects the dynamical part of polarization functions of surrounding fragments. To assess the accuracy of two approximations, monomer self-energies with the approximated screened Coulomb potential are compared with those with the exact screened Coulomb potential,

$$
\Sigma^{I}=1 / 2 \pi \int d \omega^{\prime} G^{I} W_{e x .} .
$$

Here, the screened Coulomb potential $\left(W_{e x}\right)$ is obtained from the polarization function with canonical MOs as in Eq. (4) without the fragmentation approximation. To calculate Eq. (8), the conventional DFT calculations were first performed to obtain canonical MOs, and the screened Coulomb potential was obtained from the polarization function without the fragmentation approximations. The monomer self-energies with the approximated polarization function were compared with those with the exact screened potential.

As benchmark systems, the face-to-face PEN tetramer with a spacing of $4.0 \AA$ and the PEN-C 60 dimer were considered, as shown in Fig. 1. The structure of the PEN-C 60 pair was extracted from the PEN/ $C_{60}$ bilayer heterojunction structure shown in Sec. III B. The FMO-COHSEX and FMO-GW calculations were performed with each molecule being assigned as a single independent fragment. The B3LYP was used as a starting point with the $6-31 \mathrm{G}^{*}$ basis set. The benchmark calculations were performed with ABINIT-MP software.

Table I shows the errors of HOMO, LUMO, and the HOMOLUMO (H-L) gap of the molecule in the considered systems. Here, minus signs on errors indicate that the self-energy with the approximated screened Coulomb is lower than that with the exact one.

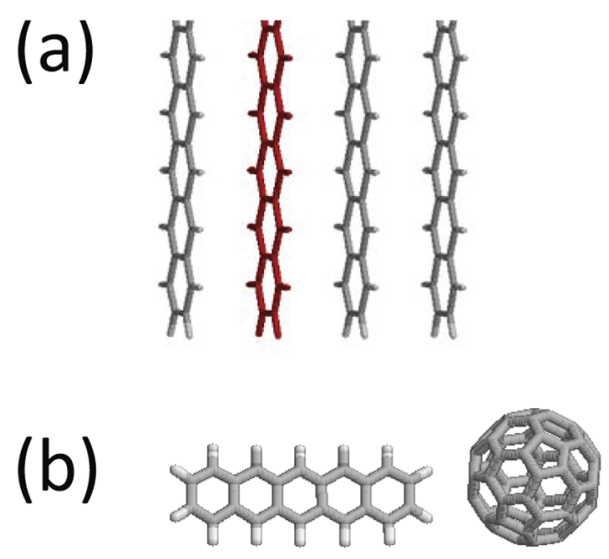

FIG. 1. Atomic structures of (a) the PEN tetramer $\left[(P E N)_{4}\right]$ and (b) the PEN- $C_{60}$ dimer. For the $(\mathrm{PEN})_{4}$ structure, the monomer self-energies were computed for the molecule depicted in red. 
TABLE I. The errors of FMO-GW self-energies in units of meV, which were obtained for HOMO, LUMO, and the HOMO-LUMO (H-L) gap.

\begin{tabular}{|c|c|c|c|c|c|c|}
\hline \multirow{3}{*}{$\begin{array}{l}\text { System } \\
\text { molecule }\end{array}$} & \multirow{2}{*}{\multicolumn{2}{|c|}{$\frac{(\mathrm{PEN})_{4}}{\mathrm{PEN}}$}} & \multicolumn{4}{|c|}{$\mathrm{PEN}-\mathrm{C}_{60}$} \\
\hline & & & \multicolumn{2}{|c|}{ PEN } & \multicolumn{2}{|l|}{$\mathrm{C}_{60}$} \\
\hline & COHSEX & GW & COHSEX & GW & COHSEX & GW \\
\hline HOMO & -8.6 & -26.3 & -8.0 & -15.2 & -6.2 & -8.4 \\
\hline LUMO & 12.1 & 6.4 & 8.3 & 16.9 & 7.1 & 7.8 \\
\hline H-L gap & 20.6 & 32.7 & 16.3 & 32.0 & 13.3 & 16.1 \\
\hline
\end{tabular}

The errors of the FMO-COHSEX solely result from the fragmentation of the polarization function. The HOMO and LUMO errors at the static COHSEX level are less than $20 \mathrm{meV}$, which is satisfactorily accurate. The overall trend is consistent with our earlier benchmark study which validates the fragmentation of the polarization function for noncovalent molecular systems. ${ }^{41}$ However, the HOMO and LUMO self-energies are lower and higher than those without the approximations, resulting in the overestimation of the $\mathrm{H}-\mathrm{L}$ gaps. This overestimation of the H-L gaps implies that the screening effects are underestimated by the fragmentation approximation.

Next, we turn to the FMO-GW self-energy errors. In addition to the fragmentation approximation at the static COHSEX level, the errors at the GW level also include the neglect of the dynamical part of polarization functions of the surrounding fragments. Overall, the GW errors are slightly more pronounced than the COHSEX errors. However, they are still acceptable, and the HOMO and LUMO selfenergy errors are less than $30 \mathrm{meV}$. The numerical results have confirmed that the two approximations employed in the FMO-GW selfenergy, the fragmentation of polarization function and combined static COHSEX and GW, can provide reasonably accurate results for noncovalent molecular systems. Although we expect that this conclusion is valid for applications to organic molecular aggregates, the FMO approximation generally deteriorates for shorter interfragment separations or for covalent systems. A more extensive assessment of the present method, as well as a possible improvement, will be investigated in a future study.

\section{B. Application to PEN/ $\mathrm{C}_{60}$ interface}

In this study, we consider the D/A bilayer heterojunction structure comprising pentacene as the electron donor and $\mathrm{C}_{60}$ as the acceptor. The $\mathrm{PEN} / \mathrm{C}_{60}$ bilayer heterojunction is one of the most prototypical organic solar cells; ${ }^{52,53}$ the electronic structures of the $\mathrm{PEN} / \mathrm{C}_{60}$ interfaces have been investigated in numerous studies, including ultraviolet photoelectron spectroscopy, ${ }^{54-56}$ external quantum efficiency (EQE) measurements, ${ }^{57,58}$ microelectrostatic calculations, ${ }^{59}$ and $a b$ initio methods. ${ }^{8,9}$ According to the $\mathrm{X}$-ray diffraction measurement, ${ }^{57}$ the $\mathrm{PEN} / \mathrm{C}_{60}$ interface morphology is dominantly an edge-on orientation. Therefore, we employed the atomic structure of the edge-on configuration of the $\mathrm{PEN} / \mathrm{C}_{60}$ bilayer heterojunction, which was prepared using molecular dynamics (MD) simulations.' Figures 2(a) and 2(b) show the total bilayer heterojunction structure and the local interface structure, (a)

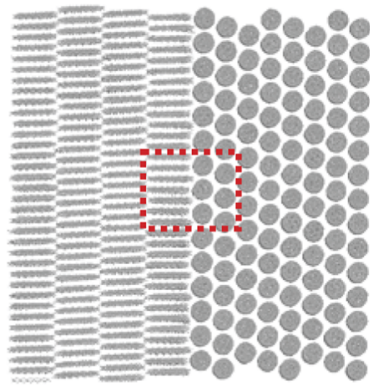

(b)

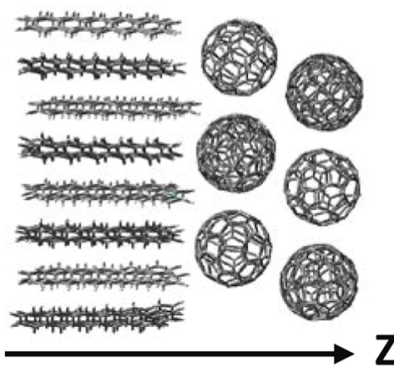

FIG. 2. (a) Atomic structure of the $P E N / C_{60}$ bilayer heterojunction and (b) the local interface structure treated by the FMO-GW calculation, which was prepared in our earlier MD simulations.

respectively. The local interface structure is composed of $36 \mathrm{PEN}$ and $15 \mathrm{C}_{60}$ molecules and thus contains 2196 atoms.

The FMO-GW calculation was performed for the local interface structure in Fig. 2(b), with the surrounding molecules in the total bilayer heterojunction being described as external point charges. In the FMO calculations, each molecule was assigned as a single independent fragment. Environmental electrostatic potentials in monomer self-consistent field (SCF) calculations were approximated by using the Mulliken point-charge approximation. ${ }^{49}$ SCF calculations for fragment dimers were not performed, since we focused on the local electronic states in which an electron (a hole) is localized within a fragment monomer. The GW quasiparticle energies and the screened Coulomb potential were computed with the B3LYP starting point and the $6-31 \mathrm{G}^{*}$ basis set. Although we note that the double-zeta quality basis set is insufficient to obtain converged self-energies, the qualitative results of the HOMO-LUMO gaps can be appropriately deduced using relatively small basis sets. ${ }^{48}$ The threshold parameter for the $\mathrm{CDAM}^{47}$ was set to be 0.01 to reduce the number of the auxiliary functions. The FMO-GW calculation was performed using the locally modified version of the ABINIT-MP program. ${ }^{49-51}$

We start our discussion by presenting the one-shot GW+BSE results for the single molecule. The corresponding GW+BSE calculation was performed for the isolated PEN or $\mathrm{C}_{60}$ molecule, whose molecular geometry was optimized at the B3LYP/6-31G ${ }^{* *}$ level using Gaussian software. ${ }^{60}$ The HOMO/LUMO quasiparticle energies were obtained as $-5.56 /-0.74 \mathrm{eV}$ for the pentacene and $-6.74 /-1.84 \mathrm{eV}$ for the $\mathrm{C}_{60}$. In Table II, the computed HOMOLUMO gap and the $S_{1}$ excitation energy (optical gap) are compared with those of the experiments. As shown, both the HOMO-LUMO and optical gaps are underestimated for the PEN by $0.21-0.38 \mathrm{eV}$, while both gaps are well reproduced for the $C_{60}$. Although the error of the PEN is marginal, the difference between the HOMO-LUMO and optical gaps, which represents the exciton binding energy, is compared well with that from the experiments. Furthermore, the difference between $\mathrm{C}_{60}$ LUMO and PEN HOMO, which is responsible for the interfacial CT energy, was calculated as $3.72 \mathrm{eV}$, which is in reasonable agreement with the experimental value of $3.91 \mathrm{eV}$. Because the CT energies are determined by the exciton binding energy and the $\mathrm{C}_{60}$ HOMO-PEN LUMO gap, the energy diagram 
TABLE II. GW HOMO-LUMO gaps and $S_{1}$ excitation energies for the isolated pentacene and $\mathrm{C}_{60}$ molecules in comparison with the experimental results.

\begin{tabular}{lccccc}
\hline \hline & \multicolumn{2}{c}{ PEN } & & \multicolumn{2}{c}{$\mathrm{C}_{60}$} \\
\cline { 2 - 3 } \cline { 5 - 6 } & $\mathrm{GW}^{\mathrm{a}}+\mathrm{BSE}^{\mathrm{b}}$ & Exp. & & $\mathrm{GW}^{\mathrm{a}}+\mathrm{BSE}^{\mathrm{b}}$ & Expt. $^{\mathrm{d}}$ \\
\hline H-L gap & 4.82 & 5.20 & & 4.90 & 4.94 \\
$S_{1}$ & 2.08 & 2.29 & & 1.71 & 1.83 \\
\hline \hline
\end{tabular}

${ }^{\text {a }}$ One-shot GW calculations with the B3LYP starting point and the $6-31 \mathrm{G}^{*}$ basis set.

${ }^{\mathrm{b}}$ BSE calculations within the Tamm-Dancoff approximation and the statically screened Coulomb potential

${ }^{c}$ References 61-63.

${ }^{\mathrm{d}}$ References 64-66.

for the charge separation can be appropriately described at the present calculation level.

We then examine the HOMO and LUMO quasiparticle energies in the PEN/C 60 interface. Figure 3 shows the HOMO and LUMO quasiparticle energies with respect to the direction perpendicular to the interface [see Fig. 2(b)]. In the vicinity of the interface, the averaged HOMO/LUMO energies are $-4.26 /-0.87 \mathrm{eV}$ for PEN molecules and $-6.12 /-2.75 \mathrm{eV}$ for $\mathrm{C}_{60}$ molecules. The $\mathrm{HOMO}$ and LUMO energies are significantly increased and decreased, respectively, compared with their gas-phase values. As a result, the HOMO-LUMO gap is reduced, known as the gap renormalization by polarizable environments. ${ }^{41,67}$ In particular, the PEN HOMO$\mathrm{C}_{60}$ LUMO gap was estimated as $1.51 \mathrm{eV}$; this is significantly reduced compared with the corresponding gas-phase value of $3.72 \mathrm{eV}$. The results have confirmed the previous findings that the induced polarization effect results in the large gap reduction from the gas to the condensed phase. $^{36,68}$

Having investigated the quasiparticle energies, we now turn to the CT states across the PEN/C 60 interface. Assuming that the CT excited state can be well described as the intermolecular HOMO-LUMO transition between the donor-acceptor molecules, we defined a CT energy from the GW quasiparticle energies and the e-h screened Coulomb potential,

$$
E_{C T}(E S+I P)=\epsilon_{G W, a}^{I}-\epsilon_{G W, i}^{J}-W_{i i, a a},
$$

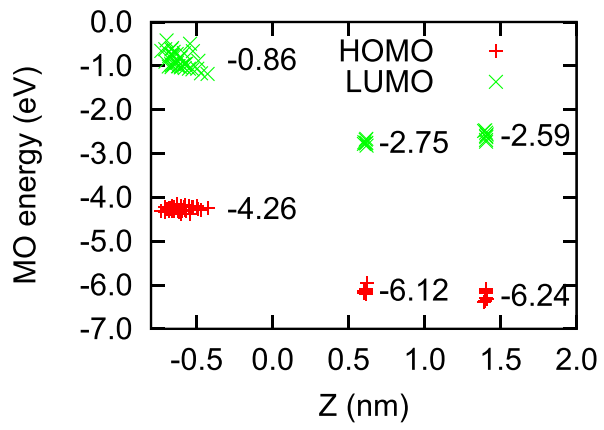

FIG. 3. FMO-GW quasiparticle energies for HOMO and LUMO of PEN or $C_{60}$ molecule in the local interface structure. where $a$ and $i$ denote the LUMO and HOMO, respectively, in this case. $W_{i i, a a}$ is the statically screened Coulomb potential,

$$
W_{i i, a a}=\int d r_{1} d r_{2} \psi_{i}^{J *}\left(r_{1}\right) \psi_{i}^{J}\left(r_{1}\right) W\left(r_{1}, r_{2}\right) \psi_{a}^{I *}\left(r_{2}\right) \psi_{a}^{I}\left(r_{2}\right),
$$

where $I$ and $J$ refer to PEN and $\mathrm{C}_{60}$, respectively. As discussed earlier, the CT energy defined by Eq. (9) includes both electrostatic (ES) and induced polarization (IP) effects labeled as "ES+IP." To separate the electrostatic and induced polarization effects, the CT energy without the environmental polarization energy was introduced as

$$
E_{C T}(E S)=\epsilon_{G W^{I}, a}^{I}-\epsilon_{G W^{J}, i}^{J}+W_{i i, a a}^{I J} .
$$

Here, the self-energy in $\epsilon_{G W^{I}, p}^{I}$ was defined by the GW selfenergy for a fragment $I$ without the environmental polarization energy $\left(\Sigma_{C O H S E X, p}^{I}-\Sigma_{C O H S E X^{I}, p}^{I}\right) ; \epsilon_{G W^{I}, p}^{I}$ does not contain the polarizability of all the other fragments including fragment $J$. The e$\mathrm{h}$ Coulomb interaction was calculated without the environmental polarization contributions, $W^{I I}=v+\int d r_{3} d r_{4} v\left(\chi_{0}^{I}+\chi_{0}^{J}\right) W^{I J}$. Therefore, the CT energy from Eq. (11) does not include the induced polarization energy associated with the formation of a CT complex.

We investigate herein the environmental effects on the CT state, taking an example of the representative state comprising a nearest-neighbor $\mathrm{PEN}-\mathrm{C}_{60}$ pair (see the supplementary material for visualization). The results of the representative $\mathrm{CT}$ state in the different environmental treatments are presented in Table III, in which the excitation energy is decomposed into the orbital-energy difference $\left(\Delta \epsilon=\epsilon_{a}^{I}-\epsilon_{i}^{J}\right)$ and the $\mathrm{e}-\mathrm{h}$ interaction $\left(E_{e-h}=-W_{i i, a a}\right)$. The results of the gas phase $\left[E_{C T}(\right.$ gas $\left.)\right]$ were obtained from the corresponding FMO-GW calculation for the isolated $\mathrm{PEN}-\mathrm{C}_{60}$ pair, which do not include any environmental effects. The bare $\mathrm{e}-\mathrm{h}$ Coulomb interaction $\left(-v_{i i, a a}\right)$ for this CT state is $-1.19 \mathrm{eV}$. The orbital-energy difference of gas-phase value $(3.18 \mathrm{eV})$ is lower than that estimated from isolated PEN and $\mathrm{C}_{60}$ molecules $(3.52 \mathrm{eV})$; the deformation of their molecular structure leads to the reduction of $\Delta \epsilon$. The magnitude of the screened Coulomb interaction is close to the $\mathrm{e}-\mathrm{h}$ bare Coulomb interaction, indicating that the static screening within the CT complex has a minor effect on the intermolecular e-h interaction. The comparison between $E_{C T}($ gas $)$ and $E_{C T}(E S)$ indicates that the environmental electrostatic effect marginally decreases the orbital-energy difference by $0.22 \mathrm{eV}$, but leaves the $\mathrm{e}-\mathrm{h}$ interaction unchanged. By contrast, the polarization effect significantly decreases the orbital-energy difference by $1.38 \mathrm{eV}$ and weakens the $\mathrm{e}-\mathrm{h}$ interaction by $0.58 \mathrm{eV}$, resulting in a decrease of the CT excitation energy by $0.89 \mathrm{eV}$. Note that the screening of e-h

TABLE III. Excitation energy, orbital-energy difference, and exciton binding energy in units of $\mathrm{eV}$ for the representative $\mathrm{CT}$ state.

\begin{tabular}{lccc}
\hline \hline & $E_{C T}$ & $\Delta \epsilon$ & $E_{e-h}$ \\
\hline Gas $^{\mathrm{a}}$ & 1.91 & 3.18 & -1.27 \\
ES $^{\mathrm{b}}$ & 1.70 & 2.96 & -1.26 \\
ES+IP & 0.91 & 1.58 & -0.68 \\
\hline \hline
\end{tabular}

${ }^{a}$ Results for the isolated $\mathrm{PEN}-\mathrm{C}_{60}$ pair.

${ }^{\mathrm{b}}$ Results without the environmental polarization energy [Eq. (11)]. 
interaction cannot be described without the induced polarization of the environment. We found that the inclusion of the environmental polarization effects is essential for computing the CT states.

Finally, we consider the energetics of the e-h separation. The energy diagram was obtained by calculating the CT states of all the PEN- $\mathrm{C}_{60}$ pairs in Fig. 2(b), where the three degenerate LUMOs of a $\mathrm{C}_{60}$ molecule were included to define the CT states. Figure 4(a) shows the CT energy as a function of the e-h separation $\left(R_{e h}\right)$, where $R_{e h}$ was defined as the center-of-mass distance between the electron and hole wave functions. ${ }^{9}$ The CT states of the nearest-neighbor $\mathrm{PEN}-\mathrm{C}_{60}$ pairs, which contribute slightly to the $\mathrm{CT}$ absorption spectrum, ${ }^{9}$ were obtained in the energy range of $0.8-1.1 \mathrm{eV}$. According to the EQE measurement of the PEN/ $C_{60}$ bilayer heterojunction, the low-energy CT absorption can be fitted to a Gaussian peak at $0.96 \mathrm{eV}$ with $0.20 \mathrm{eV}$ reorganization energy. Therefore, the CT energies calculated from the FMO-GW are in reasonable agreement with the experimental estimates. The energy landscape for the e-h separation can be characterized by the bound $\mathrm{e}-\mathrm{h}$ pairs at a low distance $\left(R_{e h}<2.0 \mathrm{~nm}\right)$ and the relatively flat energy profile $\left(R_{e h}>2.0 \mathrm{~nm}\right)$ compared with that in the earlier study.

We now clarify the impact of induced polarization on the charge separation. The induced polarization energy $E_{I P}$ of a CT state was introduced as follows:

$$
E_{I P}=E_{C T}(E S+I P)-E_{C T}(E S) .
$$

Figure 4(b) presents the induced polarization energy for the CT states, indicating that $E_{I P}$ decreases with increasing $R_{e h}$. This originates from the fact that the two separated charges are more strongly stabilized by their respective environments than a bound $\mathrm{e}-\mathrm{h}$ pair. As shown in Fig. 4(b), $E_{I P}$ decreases from $-0.7 \mathrm{eV}$ at $1.3 \mathrm{~nm}$ to
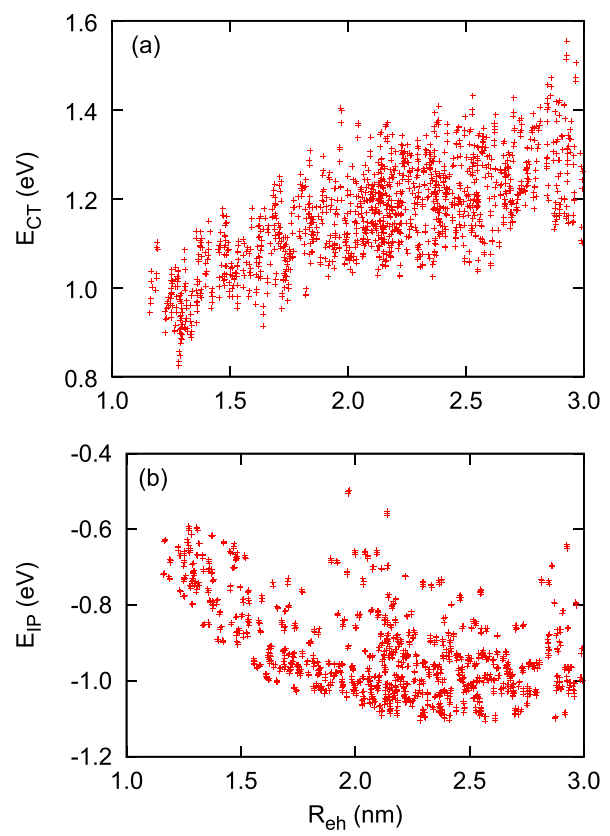

FIG. 4. (a) Energies and (b) induced polarization energies for CT states with respect to the $e-h$ separation in the $P E N / C_{60}$ interface.
$-1.1 \mathrm{eV}$ at $2.5 \mathrm{~nm}$, favoring separated charges by $\sim 0.4 \mathrm{eV}$. Although the convergence of $E_{I P}$ with respect to $R_{e h}$ might arise from the limited QM system size in our calculation, the similar convergence behavior can also be observed in the earlier microelectrostatic study by D'Avino et al. ${ }^{44}$ (see $D_{i j}^{ \pm}$of Fig. 8 in Ref. 44 ). The magnitude of the relative stabilization of the separated charges $(\sim 0.4 \mathrm{eV})$ is also comparable to their results on the $\mathrm{P} 3 \mathrm{HT} / \mathrm{C}_{60}$ interface. ${ }^{44}$ The results may support an earlier suggestion that induced polarization energy does not strongly depend on the materials.

We note that the disorder in the electronic states is a key factor for the charge separation. ${ }^{44}$ The standard deviations of the PEN HOMO and $\mathrm{C}_{60}$ LUMO are 57 and $108 \mathrm{meV}$, respectively, leading to the variation in the $\mathrm{CT}$ energies. The variations in the MO energies is due to the molecular geometries and local disorder in the electrostatic and polarizable environments. In this study, the FMOGW calculation was performed only for the local interface structure [Fig. 2(b)], and the induced polarization effects within the local structure is described. By contrast, the other molecules in the total heterojunction structure [Fig. 2(a)] were treated as the fixed point charges; the induced polarization effects from them are not incorporated. Therefore, the induced polarization effects on the molecules at the boundary between QM and MM regions are not fully taken into account, which may emphasize the disorder in the electronic states. In particular, we note from Fig. 4(b) that the induced polarization energies are not well evaluated for some of the CT states of large e-h separation, which comprise the molecules at the QM/MM boundary. The role of the disorder, as well as the asymptotic behavior of the CT states, will be clarified by employing a more extensive structure as a QM system or using a polarizable model as a MM system. $^{35,36}$

\section{CONCLUSIONS}

We have developed the fragment-based implementation of GW which enables the GW calculations for the system containing more than 2000 atoms. Our implementation is based on the fragmentation approximations for the total polarization function and combined GW and static COHSEX approximations for self-energies. In addition to the ground-state polarization, the state-specific polarization effects can be treated in the present development, allowing for descriptions of the HOMO-LUMO gap renormalization and screening of the e-h Coulomb attractions. The fragment-based GW method was demonstrated by application to the PEN/ $\mathrm{C}_{60}$ interface structure in which we have highlighted the environmental polarization effects on the CT states. The FMO-based formalism can be applied to any disordered or heterogeneous molecular aggregates and thus will offer a promising tool to explore the electronic processes.

In this article, we have considered the induced polarization effect on the localized electronic states in which an electron or a hole is localized within a single molecule. However, the electron or hole can be delocalized by intermolecular orbital interactions, and the roles of charge delocalization in the CT states have been discussed. ${ }^{70,71}$ Indeed, the delocalization of the CT states modulates the e-h energy diagram. ${ }^{7,9}$ To explore the role of delocalization, self-energy corrections to delocalized states must be calculated. In the FMO method, such delocalization effects can be recovered by constructing a total Fock matrix using fragment $\mathrm{MOs}^{72}$ 
in which the total Fock matrix is approximated from Fock matrices of fragment monomers and dimers. In the same fashion, a total self-energy matrix can be also approximated from self-energy matrices of fragment monomers and dimers. ${ }^{41}$ Indeed, the total selfenergy matrix at the static COHSEX level was considered in our earlier study. ${ }^{41}$ Although the implementation at the GW level is straightforward, this manuscript focused on the monomer states because of the larger computational cost for GW self-energies of fragment dimers. In addition, the delocalized excited states can be computed by constructing the excited-state Hamiltonian ${ }^{73,74}$ of an entire system. The combined effects of charge delocalization and state-specific polarization will be investigated in a future study.

\section{SUPPLEMENTARY MATERIAL}

See supplementary material for working equations for the FMO-GW self-energies, the visualization of the representative CT state, and the e-h energy diagram without the induced polarization energy.

\section{ACKNOWLEDGMENTS}

T.F. thanks the financial support by Building of Consortia for the Development of Human Resources in Science and Technology, MEXT, Japan and by the Grant-in-Aid for Scientific Research (C) (Grant No. 19K05255) from the Japan Society for the Promotion of Science (JSPS). T.F. also thanks Dr. Yoshio Okiyama and Dr. Tatsuya Nakano at the National Institutes of Health Science for providing the CDAM module and Professor Yuji Mochizuki at the Rikkyo University for the fruitful discussion. Y.N. acknowledges Grant-in-Aid for Scientific Research (C) (Grant No. 17K05565) from the JSPS. T.H. acknowledges Grant-in-Aid for Scientific Research (B) (Grant No. 17H02828) from the JSPS and Priority issue 7 for the Post-K computer. The majority of the FMO calculations in this work were done using the facilities of the Supercomputer Center, the Institute for Solid State Physics, the University of Tokyo. The Gaussian calculations in this work were done using Research Center for Computational Science, Okazaki, Japan.

\section{REFERENCES}

${ }^{1}$ T. M. Clarke and J. R. Durrant, Chem. Rev. 110, 6736 (2010)

${ }^{2}$ F. Gao and O. Inganas, Phys. Chem. Chem. Phys. 16, 20291 (2014).

${ }^{3}$ K. Vandewal, K. Tvingstedt, A. Gadisa, O. Inganäs, and J. V. Manca, Phys. Rev. B 81, 125204 (2010).

${ }^{4}$ J. Yao, T. Kirchartz, M. S. Vezie, M. A. Faist, W. Gong, Z. He, H. Wu, J. Troughton, T. Watson, D. Bryant et al., Phys. Rev. Appl. 4, 014020 (2015).

${ }^{5}$ Y. L. Lin, M. A. Fusella, O. V. Kozlov, X. Lin, A. Kahn, M. S. Pshenichnikov, and B. P. Rand, Adv. Funct. Mater. 26, 6489 (2016).

${ }^{6}$ H. Ma and A. Troisi, Adv. Mater. 26, 6163 (2014).

${ }^{7}$ G. D'Avino, L. Muccioli, Y. Olivier, and D. Beljonne, J. Phys. Chem. Lett. 7, 536 (2016).

${ }^{8}$ Z. Zheng, N. R. Tummala, Y.-T. Fu, V. Coropceanu, and J.-L. Brédas, ACS Appl. Mater. Interfaces 9, 18095 (2017).

${ }^{9}$ T. Fujita, M. K. Alam, and T. Hoshi, Phys. Chem. Chem. Phys. 20, 26443 (2018).

${ }^{10}$ M. Marques and E. Gross, Annu. Rev. Phys. Chem. 55, 427 (2004).

${ }^{11}$ A. Dreuw and M. Head-Gordon, Chem. Rev. 105, 4009 (2005).

${ }^{12}$ A. Dreuw, J. L. Weisman, and M. Head-Gordon, J. Chem. Phys. 119, 2943 (2003).
${ }^{13}$ R. J. Magyar and S. Tretiak, J. Chem. Theory Comput. 3, 976 (2007).

${ }^{14}$ T. Leininger, H. Stoll, H.-J. Werner, and A. Savin, Chem. Phys. Lett. 275, 151 (1997).

${ }^{15}$ H. Iikura, T. Tsuneda, T. Yanai, and K. Hirao, J. Chem. Phys. 115, 3540 (2001).

${ }^{16}$ Y. Tawada, T. Tsuneda, S. Yanagisawa, T. Yanai, and K. Hirao, J. Chem. Phys. 120, 8425 (2004).

${ }^{17}$ T. Yanai, D. P. Tew, and N. C. Handy, Chem. Phys. Lett. 393, 51 (2004).

${ }^{18}$ J.-D. Chai and M. Head-Gordon, J. Chem. Phys. 128, 084106 (2008).

${ }^{19}$ N. P. Brawand, M. Vörös, M. Govoni, and G. Galli, Phys. Rev. X 6, 041002 (2016).

${ }^{20}$ J. M. Olsen, K. Aidas, and J. Kongsted, J. Chem. Theory Comput. 6, 3721 (2010).

${ }^{21}$ N. H. List, J. M. H. Olsen, and J. Kongsted, Phys. Chem. Chem. Phys. 18, 20234 (2016).

${ }^{22}$ L. Hedin, Phys. Rev. 139, A796 (1965).

${ }^{23}$ M. S. Hybertsen and S. G. Louie, Phys. Rev. B 34, 5390 (1986).

${ }^{24}$ G. Onida, L. Reining, and A. Rubio, Rev. Mod. Phys. 74, 601 (2002).

${ }^{25}$ G. Strinati, Riv. Nuovo Cimento 11, 1 (1988).

${ }^{26}$ M. Rohlfing and S. G. Louie, Phys. Rev. B 62, 4927 (2000).

${ }^{27}$ X. Blase and C. Attaccalite, Appl. Phys. Lett. 99, 171909 (2011).

${ }^{28}$ I. Duchemin, T. Deutsch, and X. Blase, Phys. Rev. Lett. 109, 167801 (2012).

${ }^{29} \mathrm{M}$. Govoni and G. Galli, J. Chem. Theory Comput. 11, 2680 (2015).

${ }^{30}$ W. Gao, W. Xia, X. Gao, and P. Zhang, Sci. Rep. 6, 36849 (2016).

${ }^{31}$ Y. Noguchi and O. Sugino, J. Chem. Phys. 146, 144304 (2017).

${ }^{32}$ Y. Noguchi, D. Hirose, and O. Sugino, Eur. Phys. J. B 91, 125 (2018).

${ }^{33}$ B. Baumeier, M. Rohlfing, and D. Andrienko, J. Chem. Theory Comput. 10, 3104 (2014).

${ }^{34}$ I. Duchemin, D. Jacquemin, and X. Blase, J. Chem. Phys. 144, 164106 (2016).

${ }^{35}$ J. Li, G. D’Avino, I. Duchemin, D. Beljonne, and X. Blase, J. Phys. Chem. Lett. 7, 2814 (2016).

${ }^{36}$ J. Li, G. D'Avino, I. Duchemin, D. Beljonne, and X. Blase, Phys. Rev. B 97, 035108 (2018).

${ }^{37}$ J. Wehner, L. Brombacher, J. Brown, C. Junghans, O. Çaylak, Y. Khalak, P. Madhikar, G. Tirimbó, and B. Baumeier, J. Chem. Theory Comput. 14, 6253 (2018).

${ }^{38}$ M. S. Gordon, D. G. Fedorov, S. R. Pruitt, and L. V. Slipchenko, Chem. Rev. 112, 632 (2012).

${ }^{39}$ K. Raghavachari and A. Saha, Chem. Rev. 115, 5643 (2015).

${ }^{40}$ The Fragment Molecular Orbital Method: Practical Applications to Large Molecular Systems, edited by D. G. Fedorov and K. Kitaura (CRC Press, Boca Raton, FL, 2009).

${ }^{41}$ T. Fujita and Y. Noguchi, Phys. Rev. B 98, 205140 (2018).

${ }^{42}$ X. Ren, P. Rinke, V. Blum, J. Wieferink, A. Tkatchenko, A. Sanfilippo, K. Reuter, and M. Scheffler, New J. Phys. 14, 053020 (2012).

${ }^{43}$ K. Kitaura, E. Ikeo, T. Asada, T. Nakano, and M. Uebayasi, Chem. Phys. Lett. 313, 701 (1999).

${ }^{44}$ G. D’Avino, S. Mothy, L. Muccioli, C. Zannoni, L. Wang, J. Cornil, D. Beljonne, and F. Castet, J. Phys. Chem. C 117, 12981 (2013).

${ }^{45}$ K. Yamada, S. Yanagisawa, T. Koganezawa, K. Mase, N. Sato, and H. Yoshida, Phys. Rev. B 97, 245206 (2018).

${ }^{46}$ X. Blase, C. Attaccalite, and V. Olevano, Phys. Rev. B 83, 115103 (2011).

${ }^{47}$ Y. Okiyama, T. Nakano, K. Yamashita, Y. Mochizuki, N. Taguchi, and S. Tanaka, Chem. Phys. Lett. 490, 84 (2010).

${ }^{48}$ J. Wilhelm, M. Del Ben, and J. Hutter, J. Chem. Theory Comput. 12, 3623 (2016).

${ }^{49}$ T. Nakano, T. Kaminuma, T. Sato, Y. Akiyama, M. Uebayasi, and K. Kitaura, Chem. Phys. Lett. 351, 475 (2002).

${ }^{50}$ Y. Mochizuki, S. Koikegami, S. Amari, K. Segawa, K. Kitaura, and T. Nakano, Chem. Phys. Lett. 406, 283 (2005).

${ }^{51}$ S. Tanaka, Y. Mochizuki, Y. Komeiji, Y. Okiyama, and K. Fukuzawa, Phys. Chem. Chem. Phys. 16, 10310 (2014).

${ }^{52}$ S. Yoo, B. Domercq, and B. Kippelen, Appl. Phys. Lett. 85, 5427 (2004).

${ }^{53}$ S. B. Jo, H. H. Kim, H. Lee, B. Kang, S. Lee, M. Sim, M. Kim, W. H. Lee, and K. Cho, ACS Nano 9, 8206 (2015). 
${ }^{54}$ I. Salzmann, S. Duhm, R. Opitz, R. L. Johnson, J. P. Rabe, and N. Koch, J. Appl. Phys. 104, 114518 (2008).

${ }^{55}$ M. Yamamoto, Y. Nakayama, Y. Uragami, H. Kinjo, Y. Mizuno, K. Mase, K. R. Koswattage, and H. Ishii, e-J. Surf. Sci. Nanotechnol. 13, 59 (2015).

${ }^{56}$ T. Nishi, M. Kanno, M. Kuribayashi, Y. Nishida, S. Hattori, H. Kobayashi, F. von Wrochem, V. Rodin, G. Nelles, and S. Tomiya, Appl. Phys. Lett. 113, 163302 (2018).

${ }^{57}$ A. N. Brigeman, M. A. Fusella, Y. Yan, G. E. Purdum, Y.-L. Loo, B. P. Rand, and N. C. Giebink, Adv. Energy Mater. 6, 1601001 (2016).

${ }^{58}$ Y. L. Lin, F. Zhang, R. A. Kerner, T. C.-J. Yang, A. Kahn, and B. P. Rand, Appl. Phys. Lett. 112, 213302 (2018).

${ }^{59}$ S. Verlaak, D. Beljonne, D. Cheyns, C. Rolin, M. Linares, F. Castet, J. Cornil, and P. Heremans, Adv. Funct. Mater. 19, 3809 (2009).

${ }^{60}$ M. J. Frisch, G. W. Trucks, H. B. Schlegel, G. E. Scuseria, M. A. Robb, J. R. Cheeseman, G. Scalmani, V. Barone, G. A. Petersson, H. Nakatsuji et al., GAUsSian 16 Revision B.01, Gaussian, Inc., Wallingford, CT, 2016.

${ }^{61}$ V. Coropceanu, M. Malagoli, D. A. da Silva Filho, N. E. Gruhn, T. G. Bill, and J. L. Brédas, Phys. Rev. Lett. 89, 275503 (2002).

${ }^{62}$ L. Crocker, T. Wang, and P. Kebarle, J. Am. Chem. Soc. 115, 7818 (1993).

${ }^{63}$ T. M. Halasinski, D. M. Hudgins, F. Salama, L. J. Allamandola, and T. Bally, J. Phys. Chem. A 104, 7484 (2000).
${ }^{64}$ D. L. Lichtenberger, M. E. Jatcko, K. W. Nebesny, C. D. Ray, D. R. Huffman, and L. D. Lamb, MRS Proc. 206, 673 (1990).

${ }^{65}$ X.-B. Wang, H.-K. Woo, and L.-S. Wang, J. Chem. Phys. 123, 051106 (2005).

${ }^{66}$ C. Hartmann, M. Zigone, G. Martinez, E. L. Shirley, L. X. Benedict, S. G. Louie, M. S. Fuhrer, and A. Zettl, Phys. Rev. B 52, R5550 (1995).

${ }^{67}$ J. B. Neaton, M. S. Hybertsen, and S. G. Louie, Phys. Rev. Lett. 97, 216405 (2006).

${ }^{68}$ S. Sharifzadeh, A. Biller, L. Kronik, and J. B. Neaton, Phys. Rev. B 85, 125307 (2012).

${ }^{69}$ N. Sato, K. Seki, and H. Inokuchi, J. Chem. Soc., Faraday Trans. 2 77, 1621 (1981).

${ }^{70}$ B. Bernardo, D. Cheyns, B. Verreet, R. Schaller, B. Rand, and N. Giebink, Nat. Commun. 5, 3245 (2014).

${ }^{71}$ S. Gélinas, A. Rao, A. Kumar, S. L. Smith, A. W. Chin, J. Clark, T. S. van der Poll, G. C. Bazan, and R. H. Friend, Science 343, 512 (2014).

${ }^{72}$ S. Tsuneyuki, T. Kobori, K. Akagi, K. Sodeyama, K. Terakura, and H. Fukuyama, Chem. Phys. Lett. 476, 104 (2009).

${ }^{73}$ T. Fujita, S. Atahan-Evrenk, N. P. D. Sawaya, and A. Aspuru-Guzik, J. Phys. Chem. Lett. 7, 1374 (2016).

${ }^{74}$ T. Fujita and Y. Mochizuki, J. Phys. Chem. A 122, 3886 (2018). 